\title{
Factores de riesgo asociados a la aparición de alteraciones ganglionares mandibulares en bovinos
}

\author{
Costa, A. ${ }^{1}$; Arestegui, M. ${ }^{1}$; Signorini, M.L. ${ }^{2}$ \\ ${ }^{1}$ Fac. Cs. Vet., Univ. Nac. Rosario, Casilda (Santa Fe, Argentina). ${ }^{2}$ CONICET, Est.Exp. Agr. INTA, \\ Ruta 34 Km 227, Rafaela (S.Fe, Argentina). E-mail: alcosta@arnet.com.ar
}

\begin{abstract}
Resumen
Costa, A.; Arestegui, M.; Signorini, M.L.: Factores de riesgos asociados a la aparición de alteraciones ganglionares mandibulares en bovinos. Rev. Vet. 29: 2, 98-103, 2018. En los bovinos, las alteraciones ganglionares mandibulares (AGM) son afecciones con base en ganglios linfáticos de cabeza y cuello que son diagnosticadas comúnmente como actinobacilosis, tanto en las unidades productivas pecuarias como en inspecciones sanitarias oficiales en frigoríficos. Los aspectos epidemiológicos de estas anomalías son escasamente abordados por la bibliografía y se sabe muy poco acerca de los factores que se asocian con las diferentes presentaciones clínicas. El objetivo del presente trabajo fue estimar la prevalencia de AGM en establecimientos bovinos del Departamento Caseros, Provincia de Santa Fe (Argentina), e identificar los factores de riesgo asociados a su presentación. Se realizó un estudio observacional de tipo transversal mediante un muestreo en establecimientos ganaderos $(\mathrm{n}=27)$. La prevalencia estimada de AGM fue del 1,3\% en unidades productivas de base pastoril. Pudo observarse que las mayores prevalencias se asociaron significativamente o como tendencias a variables relacionadas al manejo de los animales en las unidades productivas. Los establecimientos con balance de trabajo negativo $(p=0,048)$, participación ocasional del veterinario $(p=0,090)$, antigüedad de los empleados menor a dos años $(p=0,092)$ e inadecuada condición corporal de los animales $(p=0,071)$, fueron los factores asociados a una mayor prevalencia de AGM. Estos factores implican diferentes situaciones que desfavorecen el bienestar de los animales y podrían vincularse a estrés crónico. Se destaca, además, el carácter endémico en la presentación de actinobacilosis y la necesidad de la realización de un mayor número de estudios que den cuenta de posibles tendencias estacionales en cuanto a su forma de presentación y del carácter no esporádico y posiblemente generalizado de esta afección en la región.
\end{abstract}

Palabras clave: bovino, actinobacilosis, factores asociados, manejo, epidemiología.

\begin{abstract}
Costa, A.; Arestegui, M.; Signorini, M.L.: Risk factors associated to the appearance of mandibular lymph node alterations in cattle. Rev. Vet. 29: 2, 98-103, 2018. In cattle, mandibular lymph node (MLN) alterations, located on head and neck, are commonly diagnosed as actinobacillosis in livestock production farms, as well as in sanitary inspections of slaughterhouses. The epidemiological aspects of these abnormalities are rarely approached in literature and little is known about the factors associated to their various clinical manifestations. The objectives of this work were to assess the prevalence of MLN in cattle farms from Caseros District in Santa Fe Province (Argentina) and to identify the risk factors associated to its presentation. An observational, cross-sectional study was carried out by sampling livestock farms $(\mathrm{n}=27)$. A $1.3 \%$ prevalence was detected in productive units under grazing systems. The higher prevalence observed was significantly associated or showed tendencies to variables related to animal management in the farms. A higher MLN prevalence was associated to factors such as negative work balance $(p=0.048)$, irregular veterinarian visits $(p=0.090)$, operators with less than two years of experience $(p=0.092)$ and animals with poor body condition in farms $(p=0.071)$. These factors involve different situations which act against animal welfare and may be linked to chronic stress. This work also raises the discussion about the endemic nature of actinobacillosis and the need of more studies that account for the possible seasonal tendencies, in relation to its form of manifestation and to the non-sporadic and possibly widespread nature of this condition in the region.
\end{abstract}

Key words: cattle, actinobacillosis, associate factors, handling, epidemiology. 


\section{INTRODUCCIÓN}

La actinobacilosis es una enfermedad infecciosa crónica, que afecta tejidos blandos, principalmente en cabeza y cuello, produciendo linfadenitis piogranulomatosa y evolucionando generalmente con abscedación de los linfonódulos regionales de cabeza y cuello ${ }^{4}$, pudiendo ocurrir también en piel, pulmones y pre-estómagos ${ }^{12}$. El ganado vacuno es principalmente afectado, pero puede involucrar a otras especies ${ }^{4} \mathrm{y}$ es considerada de presentación esporádica $7,11,12$.

Sin embargo, datos de decomisos en frigoríficos a nivel regional y local, sugieren que esta enfermedad supera las presentaciones esporádicas ${ }^{3,8}$. A partir de la descripción en Argentina de la actinobacilosis bovina en $1902{ }^{6}$ se ha generado información sobre el agente causal y el proceso patológico, pero poco se conoce sobre los factores que se asocian a su presentación.

El presente trabajo abordó la situación epidemiológica de las alteraciones ganglionares mandibulares (AGM), comúnmente diagnosticadas como actinobacilosis, con la intención de aportar información científica que pudiera ser empleada como base para la elaboración de programas de prevención y control de estas alteraciones.

El objetivo de este trabajo fue estimar la prevalencia e identificar los factores de riesgo asociados a la presentación de las alteraciones ganglionares mandibulares en bovinos del Departamento Caseros, Provincia de Santa Fe, Argentina.

\section{MATERIAL Y MÉTODOS}

Diseño del estudio observacional. Se realizó un estudio observacional de tipo transversal, mediante un muestreo en establecimientos ganaderos del departamento mencionado ut supra. El mismo contaba con un stock ganadero mayor de 72.000 bovinos (dato de 2010). Dada la inexistencia de antecedentes que permitan precisar la prevalencia y distribución de casos de AGM, se procuró obtener información a partir de unidades productivas de los distintos distritos del departamento. El muestreo coincidió con la vacunación antiaftosa sistemática del año 2012 en cada unidad productiva.

La obtención de datos se efectuó a partir de indagaciones procedentes de fuentes primarias y secundarias. En cada establecimiento se aplicó una encuesta estructurada dividida en secciones con características generales del establecimiento y potenciales factores asociados a la presentación de AGM, que fueron consideradas como "variables independientes". El propósito y la importancia de dicha encuesta fue explicada previamente enfatizando que las respuestas eran anónimas y que el interés de la misma estaba dirigido a indagar frecuencia de eventos a nivel poblacional.

El cuestionario abarcó 10 planillas con parámetros descriptivos de las unidades productivas y preguntas con opciones de respuestas estructuradas. Fue respondido por el veterinario actuante, con participación del productor o encargado en el $48 \%$ de los casos, por el productor en el $40 \%$ y por el encargado en el $12 \%$ restante. Los datos se cargaron en planilla electrónica. Las fuentes secundarias fueron obtenidas del Servicio Nacional de Sanidad Agroalimentaria (SENASA).

Estimando una prevalencia de AGM del $1 \%$, una confianza del $95 \%$ y un error estimado del $4 \%$, se decidió muestrear un mínimo de 25 establecimientos totales en el departamento, estratificados de acuerdo a tipo de explotación ganadera y cantidad de bovinos. El muestro se amplió finalmente a 27 establecimientos, que incluyeron tambos (7), cría e invernada (13) y feed- lots (7). De acuerdo a la cantidad de animales por establecimiento siete de ellos tenían hasta 50 bovinos, otros siete poseían de 51 a 100 y los trece restantes más de 100 .

En cada establecimiento el proceso implicó el encierre en corrales y posterior conducción por brete o manga de todos los bovinos existentes en el rodeo, situación que permitió la inspección, detección y separación de los animales que presentaron alteraciones ganglionares mandibulares.

Identificación de variables. La variable dependiente del estudio fue la presencia o ausencia de casos de bovinos que presentaron AGM. Estos "casos" se definieron, para los fines del presente trabajo, como abscesos y lesiones granulomatosas de ganglios linfáticos mandibulares, subparotídeos, retrofaríngeos y tejidos blandos de cabeza y cuello, con presentaciones que abarcaron casos activos, con aumento de tamaño, registrable por inspección, con o sin presencia de exudados y fistulizaciones.

En algunos casos se sumaron nódulos y abscesos satélites. El exudado producto del proceso fue espeso, de alta cohesión y de color amarillento. Pudieron evolucionar con deformaciones residuales, caracterizadas por alteraciones en forma y tamaño sin signos de inflamación aguda. Las variables independientes surgieron a partir de la encuesta aplicada a los productores visitados, las cuales se detallan a continuación.

Las actividades productivas se agruparon de acuerdo a tres especializaciones: cría e invernada $(\mathrm{n}=13)$, tambo $(\mathrm{n}=7)$ y feed-lot $(\mathrm{n}=7)$. Forma de pastoreo: se relevaron tres formas diferentes de pastoreo: continuo, rotativo y rotativo con alta carga Suplementación proteica: se identificaron las unidades productivas que emplearon concentrados proteicos y aquellas que no utilizaron este insumo. Suplementación energética: se identificaron aquellos establecimientos que la emplearon y los que no la emplearon. Suplementación con heno y suplementación con silo: la inclusión de estas variables fue considerada relevante dado que el heno, por su característica fibrosa, puede ser injuriante para la mucosa oral del bovino y favorecer la presencia de enfermedad ${ }^{4,11}$.

Destete y manejo sanitario. En el área de estudio se caracterizaron dos tipos de procesos de destete para el análisis: destete abrupto y destete variable. Para la 
participación del médico veterinario se establecieron dos categorías: incorporado, cuando el profesional participaba del manejo del establecimiento, entendiéndose esta situación como la articulación de recursos con el fin productivo; y ocasional, cuando participaba solo en la actividad sanitaria.

Para el manejo sanitario se identificaron dos categorías: a) básico, que implicó la realización de las acciones sanitarias obligatorias, quedando las situaciones contingentes a cargo de un profesional distinto al co-responsable sanitario o directamente a cargo del productor; y b) programado que implicó la realización de las acciones sanitarias obligatorias y el resto de medidas relacionadas a la salud y producción del rodeo, por parte del co-responsable sanitario, enmarcado en la planificación acordada con el productor.

Balance de trabajo. A los efectos de disponer de una variable que refiera a la organización del trabajo con los animales en cada unidad productiva, se empleó una adaptación de la metodología denominada balance de trabajo o bilan travail ${ }^{2}$. Se trata de una herramienta para la comprensión de la organización y dinámica del trabajo en explotaciones agropecuarias. Se seleccionó como "tarea" a la inspección de los animales, que en terminología pecuaria es la "recorrida".

Esta tarea es relevante porque mediante su correcta realización se verifica el estado general de los animales. Además, no es captada por otras variables relevadas como cantidad de personal o antigüedad del mismo. Para determinar el valor positivo o negativo de esta variable se procedió de la siguiente manera: los casos que registraron limitaciones tales como ausencia de personal permanente en el predio o cuando el indicador resultó mayor o igual a 1 (frecuencia de realización $>$ a una semana) se consideraron negativos (balance de trabajo negativo).

Por el contrario, cuando el indicador "frecuencia de realización" fue menor a 1, el balance de trabajo fue positivo. Este resultado pudo variar y considerarse negativo cuando se registraron restricciones. Se consideraron restricciones: a) dificultades para la implementación del manejo o tratamientos de los animales por escasez de instalaciones, alambrados ausentes o en mal estado, conducción de los animales desde el lote hasta las instalaciones de trabajo dificultada por excesiva distancia o interposición de áreas agrícolas sin alambrar; y b) desatención de los animales por el inadecuado resultado económico de la actividad.

Condiciones de los animales. Como condición corporal se empleó una escala de 5 puntos, con puntuaciones intermedias. Por su parte, las categorias animales fueron establecidas de acuerdo al acta de vacunación. En cuanto al biotipo, los bovinos presentes en las diferentes unidades productivas se agruparon en dos grandes categorías: Holstein y otros. Esta última incluyó cruzas de razas europeas.
Cantidad y antigüedad del personal. Se clasificó a los establecimientos según la cantidad de personal para el trabajo con el ganado según la existencia de menos de dos y de dos o más personas. Con respecto a la antigüedad, de acuerdo al artículo 143 del código sustantivo del trabajo, la antigüedad en un puesto ha servido para argumentar que a mayor tiempo y experiencia desempeñando un oficio, se logra mayor eficiencia. Con este criterio, se clasificó a los establecimientos según la antigüedad promedio de sus empleados en menor a dos años y en mayor o igual a dos años.

Análisis estadísticos. Para cuantificar el riesgo asociado a la presencia de AGM en los bovinos (presencia/ ausencia), se realizó un análisis en dos etapas. Inicialmente, todas las variables fueron comparadas con la variable dependiente (análisis bivariado) empleando el test T-Student, prueba de Mann-Whitney, $\chi^{2}$ o test exacto de Fisher, de acuerdo a las características de la variable independiente. Posteriormente se realizó una regresión logística (análisis multivariante), incluyendo en el modelo aquellas variables que hubieran estado asociadas con la variable dependiente en el análisis bivariado con una significancia (p) inferior a 0,15 .

El método empleado fue un modelo generalizado lineal mixto, con los establecimientos como variable aleatoria. La existencia de asociaciones entre las variables independientes fue evaluada mediante el cálculo de la correlación de Spearman (r). Cuando dos potenciales factores de riesgo estuvieron asociados $(r>0,6)$, solamente se incluyó en el análisis multivariante aquella con menor valor de significancia en el análisis bivariado. Todos los análisis estadísticos se realizaron empleando el paquete estadístico InfoStat (Universidad Nacional de Córdoba, Argentina).

\section{RESULTADOS}

Estimación de la prevalencia de AGM. Se examinaron 2.161 bovinos presentes en 20 unidades productivas de cría, invernada y tambo, encontrándose 29 casos positivos de AGM, resultando una prevalencia del 1,3\%. También se examinaron 5.814 bovinos de 7 establecimientos de feed-lot encontrándose solo 7 casos positivos, resultando una prevalencia del $0,14 \%$.

Dado el bajo valor de prevalencia hallado en los establecimientos de feed-lot y las características resueltas en la sustitución por tejido conectivo de los abscesos de los animales positivos, esta categoría no se incluyó en el estudio. Al encontrarse en los feed-lots un número muy importante de animales y con baja prevalencia de AGM, si se hubieran incorporado a los análisis habrían generado asociaciones espurias.

Los análisis estadísticos de regresión o correlación están sumamente influenciados por el número de unidades incorporadas al análisis. De esta forma, una cantidad muy elevada de animales y una baja prevalencia de la enfermedad, provocarían que la totalidad de las variables independientes resulten estar asociadas esta- 
dísticamente con la aparición de AGM, aunque muchas de ellas serian espurias.

Esta misma desproporción entre casos de AGM y animales totales incorporados al estudio, invalidaría la posibilidad de incorporar todas las variables significativas en un análisis bivariado a un modelo de regresión logística, generando coeficientes de regresión desvirtuados.

Análisis divariado. La prevalencia de AGM no estuvo influenciada por actividad productiva $(p=0,737)$, categoría de los animales $(p=0,213)$, componente forrajero $(p=0,228)$, suplementación con heno $(p=0,367)$ o silajes $(p=0,367)$, ni por el biotipo de los animales $(p=0,194)$. No obstante, la forma de pastoreo estuvo relacionada con la probabilidad de encontrar esta patología. Establecimientos cuyos animales pastoreaban de manera continua tuvieron mayores probabilidades de presentar AGM que aquellos que practicaban pastoreo rotativo $(p=0,063)$.

Tanto la suplementación energética $(p=0,044)$ como la proteica $(p=0,007)$ estuvieron asociadas a una reducción en la probabilidad de de presentación de AGM y esto fue más evidente cuando los animales fueron suplementados tanto con energía como con proteínas $(p=0,016)$. Con respecto a los veterinarios, cuando estos participaban de manera ocasional, la prevalencia de AGM fue superior a la observada en las explotaciones en las cuales ellos estaban incorporados de manera estable $(p=0,004)$.

De igual forma, cuando el manejo sanitario fue básico, la prevalencia de AGM fue superior a la hallada en explotaciones con un manejo sanitario programado $(p=0,047)$. Las explotaciones con menos de dos empleados tendieron a tener mayores prevalencias de AGM que aquellas con mayor dotación de personal $(p=0,070)$. De igual forma, cuando los trabajadores tuvieron más de 2 años de trabajo las prevalencias de AGM tendieron a ser menores en comparación con las explotaciones que contaban con empleados de menor antigüedad laboral $(p=0,030)$.

Finalmente, cuando el balance de trabajo fue positivo, las prevalencias de AGM tendieron a ser significativamente menores en comparación con los establecimientos con balance de trabajo negativo $(p<0,001)$. Los animales cuyo destete fue realizado de manera abrupta mostraron mayor tendencia a presentar AGM que aquellos animales que fueron sometidos a un destete variable $(p=0,097)$. Finalmente, la condición corporal de los animales fue otro factor asociado con la presencia de AGM, dado que las prevalencias tendieron a incrementarse en animales con inadecuada condición corporal $(p=0,122)$.

Análisis multivariante. De acuerdo al modelo generalizado lineal mixto, la única variable asociada estadísticamente con la presencia de AGM a un nivel de significancia $<0,05$ fue el balance de trabajo. Aquellos establecimientos con balance de trabajo negativo tuvieron en promedio 7,306 veces más riesgo (odds ratio) de presentar AGM que las explotaciones con balance de trabajo positivo $(p=0,048)$.

Otras variables presentaron valores de significancia $<0,1$ y pueden ser consideradas como tendencias. En este sentido, en aquellos establecimientos cuyos empleados tenían menor experiencia laboral $(<2$ años) los bovinos tuvieron 4,119 veces más riesgo de tener AGM $(p=0,092)$ en comparación con los bovinos de explotaciones cuyos empleados tenían mayor experiencia.

Cuando el veterinario participaba de manera ocasional en el manejo sanitario de los rodeos, los vacunos tuvieron 5,217 veces más riesgo $(p=0,092)$ de presentar AGM que en los casos donde el profesional estaba incorporado al trabajo del establecimiento.

Finalmente, los bovinos con mala condición corporal tuvieron 5,122 veces más riesgo de padecer AGM $(p=0,071)$ que aquellos con adecuada condición corporal. Las demás variables presentaron valores de significación $>0,1$ lo que significa que no mostraron una asociación significativa con la aparición de AGM, aunque pudieran estar ligadas de manera indirecta con la presentación de alguno de los factores de riesgo.

\section{DISCUSIÓN}

El resultado de prevalencia obtenido es un dato puntual, no obstante (en función de la información bibliográfica y los datos anamnésicos obtenidos durante el presente estudio) es posible discutir el carácter esporádico de las AGM en el área de estudio. En todos los establecimientos se reconoció la afección como persistente y crónica.

En Argentina la información sobre registros de enfermedades en bovinos es limitada, abarcando las de denuncia obligatoria y aquellas identificadas durante la inspección post-mortem en frigorífico. Sin embargo, estudios específicos en diferentes regiones del país refuerzan la idea de la persistencia del problema.

Un estudio de prevalencia realizado por el autor en un distrito de la Provincia de Santa Fe, arrojó una prevalencia de 0,66\% $(n=10980)$. También, en la Provincia de La Pampa, se obtuvo una prevalencia de actinobacilosis y actinomicosis del $2,51 \%{ }^{8}$. De esta manera, los estudios realizados en diferentes momentos, sobre poblaciones bovinas en lugares distintos, son consistentes con los resultados del presente trabajo, sugiriendo la presencia endémica de la enfermedad, aunque con baja prevalencia.

El balance de trabajo negativo fue la única variable asociada estadísticamente con la presencia de AGM. La construcción de esta variable captó tanto cuestiones técnicas como de manejo, y muestra cómo la forma en que se organiza el trabajo en un establecimiento puede influir en la salud de los animales. Su fuerza explicativa superó la de variables estrictamente biológicas en función que la variable balance de trabajo se relaciona con el manejo de la unidad productiva y es un componente de mayor jerarquía en el sistema. Esto significa que componentes biológicos del sistema dependen del 
manejo, entendido este como la articulación de recursos que con ellos se realice.

Otras tres variables presentaron valores de significancia $<0,1$ y pueden ser consideradas como tendencias. Estas fueron: condición corporal inadecuada ( $p=$ $0,071)$, participación veterinaria ocasional en el manejo sanitario de los rodeos, $(p=0,092)$ y antigüedad del personal menor a dos años $(p=0,092)$.

En algunas situaciones se ha estudiado la condición corporal como manifestación del efecto de ciertas enfermedades ${ }^{9}$. Tal criterio no pude extenderse a nuestro estudio, donde la inadecuada condición corporal estuvo asociada a variables alimenticias y nutricionales que afectaron generalizadamente a los bovinos. Por lo tanto, en nuestro caso, la condición corporal inadecuada puede considerarse preexistente a la enfermedad. La condición corporal captó la variabilidad de tipo de pastoreo, ya que el pastoreo continuo implicó una alimentación con base en pasturas naturales y su lógica fluctuación estacional genera gran variación en la disponibilidad de nutrientes, situación relacionada a condición corporal inadecuada.

De un modo similar, el $16,3 \%$ de los animales que se destetaron de manera abrupta tuvieron una inadecuada condición corporal, mientras que ninguno de los animales destetados de manera variable presentó una condición corporal inadecuada $(p<0,001)$. El destete abrupto, dado que su forma de realización no tiene en cuenta los requerimientos del ternero, puede provocar un estado de subnutrición, con relación a los terneros amamantados al pie de madre. Este estado puede afectar la salud y producción animal ${ }^{1}$.

La ausencia de suplementación fue otra variable que, presentándose asociada en el análisis bivariado, no lo estuvo en el multivariante. Esta variable presentó una lógica relación con la condición corporal de los bovinos afectados. Cuando los animales no recibieron suplementación proteica, aproximadamente el 19\% padeció una inadecuada condición corporal, mientras que todos los animales que recibieron suplementación proteica mostraron una adecuada condición corporal $(p<0,001)$. Dietas con un contenido menor a 6-8\% de proteína bruta, como puede ocurrir en ciertos períodos del año en el pastoreo continuo, serían limitantes para los microorganismos ruminales y por lo tanto para la salud de los animales ${ }^{10,12}$.

Los establecimientos que contaron con participación veterinaria ocasional mostraron restricciones en la aplicación de aspectos técnicos. Tuvieron tendencia a no suplementar energética y/o proteicamente a los animales $(p<0,001)$, el manejo sanitario fue básico $(p<0,001)$, presentaron destete abrupto $(p<0,001)$ y realizaban pastoreo continuo $(p<0,001)$. Todas estas variables, que estuvieron asociadas a la variable respuesta en el análisis bivariado, resultaron no estar asociadas en el multivariante. No obstante, estas variables estuvieron condicionadas por la participación del veterinario, variable considerada como factor de riesgo de la aparición de AGM.
La actividad del veterinario, cuando se desarrolló de manera incorporada al manejo del establecimiento, repercutió positivamente registrándose menos casos de actinobacilosis y seguramente también en otros parámetros de salud que escapan al presente estudio. La antigüedad de los empleados de las unidades productivas también puede ser considerada una tendencia: antigüedad del personal $<$ a 2 años captó la variabilidad del tipo destete $(p<0,001)$ y la forma en la cual pastoreaban los animales $(p<0,001)$. Establecimientos que contaban con personal de poca antigüedad ( $<$ a 2 años) tendieron a destetar de manera abrupta y pastorear de forma continua. Esta relación es lógica y permite discutir sobre la problemática del trabajo rural, donde las oportunidades de capacitación formal son escasas.

Claramente, los factores asociados con las presentaciones clínicas son la expresión negativa de las variables estudiadas y sugieren en todos los casos situaciones que pueden desfavorecer el bienestar de los animales y podrían en algunos casos vincularse a situaciones de estrés crónico. Las características de este estudio limitan la posibilidad de concluir sobre esta relación pero existen referencias en tal sentido y se considera probable que la situación contribuya en esas presentaciones ${ }^{4,5}$.

El abordaje epidemiológico en este estudio nos permite proponer que la corrección de la expresión de las variables identificadas, es necesaria para lograr la reducción de la prevalencia de AGM en el área de estudio. Es importante destacar que, para mejorar cualquier aspecto de la salud en un conjunto de animales, deben atenderse las cuestiones intrínsecas del proceso patológico. Pero también es probable que el problema no se resuelva si no se modifican factores restrictivos externos al animal como los analizados en este trabajo. Esto impulsa a medir fenómenos complejos y analizarlos rigurosamente, como contribución para estar más cerca de resolver problemas sanitarios.

Puede concluirse que las AGM se presentan, en el área bajo estudio, en forma endémica y con bajas prevalencias. Las mayores prevalencias se asociaron a variables relacionadas al manejo de los animales en las unidades productivas, tal es el caso del balance de trabajo (negativo), el tipo de participación del veterinario (ocasional), la experiencia laboral de los empleados (menor a dos años) y la condición corporal de los animales (inadecuada). Las expresiones negativas de las variables estudiadas en las unidades productivas con presencia de enfermedad, sugieren en todos los casos situaciones que desfavorecen el bienestar de los animales. La corrección de la expresión de las variables identificadas es necesaria para lograr la reducción de la prevalencia de AGM en el área de estudio.

\section{REFERENCIAS}

1. Coppo JA. 2007. ¿El destete precoz produce estrés en los terneros cruza cebú? Rev Electr Vet (Redvet, España) 8: $14-49$. 
2. Dedieu B, Laurent C, Mundler P. 1999. Organisation du travail dans les systèmes d'activités complexes. Économ Rurale 253: 28-35.

3. Dubarry JR et al. 2004. Actinomicosis y actinobacilosis: una causa frecuente de lesiones granulomatosas en bovinos de Maracó (La Pampa, Argentina). Ciencia Vet 6: 3738.

4. Jubb KV, Kennedy PC, Palmer N. 2008. Pathology of domestic animals, $5^{\text {th }}$ ed., Elsevier-Saunders, Edinburgh, p. 2-20.

5. Kazufumi K, Tilusha M, Kei U, Sayaka K, Kou T, Naoto I, Tomoyuki S. 2017. Multifocal suppurative granuloma caused by Actinobacillus lignieresii in the peritoneum of a beef steer. The J Vet Med Sci 79: 65-67.

6. Lignieres J, Spitz G. 1902. L'actinobacilose. Bull Soc Centr Med Vet 20: 487-536.

7. Manual Merck de Veterinaria. 2007. Océano/Centrum, $6^{\mathrm{a}}$ ed., Barcelona, p. 464.
8. Miranda AO. 2014. Epidemiology of cattle diseases in the northeast of La Pampa, Argentina. Rev Vet 25: 81-86.

9. Morales G. 2012. Relación entre la condición corporal y el nivel de infestación parasitaria en bovinos a pastoreo como criterio para el tratamiento antihelmíntico selectivo. Rev Inv Vet Perú 23: 80-89.

10. Noro M, Vargas V, Pulido R, Wittwer F. 2006. Efecto del tipo de concentrado sobre indicadores sanguíneos del metabolismo de energía y de proteínas en vacas lecheras en pastoreo primaveral. Arch Med Vet 38: 227-228.

11. Radostits OM, Gay CC, Blood DC, Hinchcliff KW. 2007. Veterinary Medicine, 10th ed., Saunders-Elsevier, London, p. 1044-1048.

12. Rycroft A, Garside L. 2000. Actinobacillus species and their role in animal disease. The Vet $J 159$ : 18-36.

\section{Revista Veterinaria ingresa a SciELO}

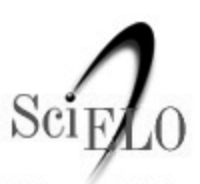

Scientific Electronic Library Online

Revista Veterinaria, publicación oficial de la Facultad de Ciencias Veterinarias de la Universidad Nacional del Nordeste (Corrientes, Argentina), ha logrado acceder al Núcleo Básico de Revistas Científicas Argentinas (Nivel 1), luego de calificar adecuadamente en el Centro Argentino de Información Científica y Tecnológica (CAICYT), según Resolución 2485/14 del Consejo Nacional de Investigaciones Científicas y Técnicas (CONICET).

Sobre un puntaje máximo de 33 se obtuvieron 32 puntos. Tal calificación constituye "una garantía de la excelencia de la publicación" (sic) y queda expedita la vía del Portal SciELO (Scientific Electronic Library Online) para los artículos publicados.

En tal calificación gravitó positivamente la circunstancia de haber aumentado el índice de impacto (Scimago-Elsevier) y haber disminuido las autocitaciones. También se tuvieron en cuenta aspectos como la amplia cobertura de la revista, la calidad científica del Comité Editorial, los criterios de evaluación de los artículos, el origen de los autores (locales $60 \%$, nacionales $13 \%$, extranjeros $27 \%$, en idioma inglés), el adecuado balance entre trabajos científicos originales y reseñas bibliográficas (ambos con alta calidad), así como el estricto cumplimiento de la periodicidad semestral y la favorable acogida por indizadores como Cab, J-Gate, Doaj, Ebsco, Gale Cengage, Infocyt, Latindex y Scopus.

Se consolida de esta manera la continuidad de "Revista Veterinaria", que en su acontecer registra más de 50 años de existencia en nuestra Facultad de Ciencias Veterinarias, entidad que en 2018 cumplió el $98^{\circ}$ aniversario de su fundación. 